\title{
Alteration of $\beta$-secretase traffic by the receptor tyrosine kinase signaling pathway - a new mechanism for regulating Alzheimer's $\beta$-amyloid production
}

\author{
Yun-Wu Zhang ${ }^{1}$, Huaxi $\mathrm{Xu}^{1}$ \\ ${ }^{1}$ Center for Neuroscience and Aging, Burnham Institute for Medical Research, 10901 N. Torrey Pines Rd., La Jolla, CA 92037, USA. \\ xuh@burnham.org
}

Cell Research (2007) 17:389-401. doi:10.1038/cr.2007.41; published online 14 May 2007

The receptor tyrosine kinases (RTKs) are a family of cell surface proteins with diverse functions in proliferation, differentiation or cell-cell communication. When a specific ligand binds to its cognate receptor, a conformational change of this receptor due to the ligand-receptor interaction will lead to activation of the intrinsic tyrosine kinase residing in the intracellular domain of the receptor. The activation of this tyrosine kinase is essential for transducing the signals to a cascade of its downstream molecules that eventually cause related physiological responses [1]. For example, binding of nerve growth factor (NGF) to its receptor TrkA is essential for the proper development, patterning, and maintenance of the mammalian nervous system. This ligand and receptor interaction will lead to the formation of a crab-shaped homodimeric TrkA structure [2], and the subsequent activation of its intrinsic RTK will cause auto-phosphorylation of its own intracellular tyrosine residues. Phosphorylated TrkA receptors recruit and increase the phosphorylation of PLC- $\gamma$ and Shc, which leads to activation of either the PI3K/Akt pathway or Ras/raf/ERK pathway. In the brain of Alzheimer's disease (AD) patients, alterations of nerve growth factor (NGF) and its receptor TrkA have been reported to associate with $\mathrm{AD}$ pathogenesis [3]. However, the underlying mechanisms remain elusive.

The presence of extracellular senile plaques in the brain is a key pathologic feature of AD. The major components of the senile plaques are small peptides called $\beta$-amyloid $(A \beta)$; overproduction of $A \beta$ is widely believed to initiate a cascade of neurodegenerative events that lead to deposition of $A \beta$, neurofibrillary tangles, and neuronal loss [4]. $A \beta$ is produced through sequential cleavages of its precursor protein APP by $\beta$-secretase (BACE1) and $\gamma$-secretase.
Alternatively, APP on the cell surface can be cleaved by $\alpha$-secretase within the $A \beta$ domain to release non-amyloidogenic soluble APP $\alpha$, which is neuroprotective [5]. BACE1 is a membrane bound aspartyl protease that preferentially cleaves its APP substrate in a relatively acidic environment. Genetic deletion of BACE1 almost completely abrogates production of $\mathrm{A} \beta$, confirming that BACE1 is a primary $\beta$-secretase [4]. The $\gamma$-secretase is composed of at least four transmembrane proteins: presenilin 1 or 2, nicastrin, APH-1 and PEN-2. Genetic deletion of any component of the $\gamma$-secretase complex abolishes the processing of APP at the $\gamma$-secretase site [4]. Moreover, genetic mutations in genes encoding APP, presenilin 1 or 2 in humans have been linked to the early onset of AD. Hence, knowledge of cellular regulation of these secretases, including regulation of their subcellular localizations, is important in the development of AD therapy.

Previous studies have suggested that RTK activation may contribute to $\mathrm{AD}$ pathogenesis by affecting secretases responsible for $A \beta$ generation [5]. In this issue of Cell Research, Pei and colleagues have further made a mechanistic link of RTK activation to the regulation of processing of APP in releasing $A \beta[6]$. In both their in vitro and in vivo studies, Pei and colleagues demonstrate that activation of NGF or EGF receptor pathways specifically elevates BACE1 activity. BACE1 is a membrane bound aspartyl protease that is a primary $\beta$-secretase as confirmed by studies with BACE1 null mice. Increased BACE1 activity has been demonstrated to result in increased production of $A \beta$. Although BACE1 is largely localized in the Golgi/TGN (trans-Golgi network) and only a small part of it is on the cell surface [7], it appears that the internalization of this 
small portion of BACE1 is important in the production of $A \beta$. The authors show that activation of RTKs by EGF or NGF enhances internalization of BACE1 to the endosomes and Golgi/TGN and facilitates the production and secretion of $A \beta$. If the Rab5-mediated early endosomal pathway is blocked, the internalization of BACE1 upon activation of RTKs by EGF or NGF is prevented. Together these results suggest that $\mathrm{NGF}$ and $\mathrm{EGF}$ can regulate $\mathrm{BACE} 1$ activity and $A \beta$ production through the regulation of BACE1 trafficking.

As the TGN and the endosomes provide a relatively more acidic environment optimal for BACE1 activity, an enhanced internalization of BACE1 into the two compartments upon RTK activation may likely contribute to an increased production of $A \beta$. Interestingly, recent studies have found that the reversible phosphorylation of BACE1 on Ser-498 near the C-terminus by casein kinase I regulates BACE1 localization between TGN and endocytotic compartments but not on the cell surface [8-9]. Hence the scenario whereby RTKs mediate activation of Ser/Thr kinases, which in turn phosphorylate BACE1 resulting in a change in $A \beta$ generation, awaits further scrutiny. Furthermore, intracellular trafficking of APP can be regulated by a variety of signaling compounds or pathways such as PKA, PKC, estrogen and insulin/EGF receptor pathways [5]. Thus it would be interesting to investigate whether RTK activation can also affect the internalization of APP, the substrate of BACE1. Such a convergence may explain the increased production of $\mathrm{A} \beta$ because of the concomitantly increased presence of APP and BACE1 in the same compartments. Abnormal intracellular trafficking of the protein components required for the generation of $A \beta$ can contribute to $\mathrm{AD}$ pathogenesis $[5,10]$. The work by Pei and colleagues has added another intriguing example. While the new discovery is appealing, it is still unclear how activation of RTKs can lead to the increased internalization of BACE1. Lastly, recent studies have demonstrated that BACE1 cleaves several other proteins besides APP, such as sialyltransferase ST6Gal I, the adhesion protein P-selectin glycoprotein ligand-1 (PSGL-1), $\beta$-subunits of voltage-gated sodium channels, and neuregulin 1 , which accounts for some of the detrimental side-effects in the
BACE1 KO animal models, noticeably at the late stage of life. An immediate question would therefore be whether the RTK-mediated change in BACE1 compartmentalization and activity is more specific to APP than to the other substrates. Identifying a novel approach to inhibit BACE1's enzymatic activity more specifically on APP processing than on the cleavage of other substrates would represent a superior therapeutic intervention against this devastating disease.

\section{References}

1 Hupfeld CJ, Olefsky JM. Regulation of receptor tyrosine kinase signaling by GRKs and $\beta$-arrestins. Annu Rev Physiol 2007; 69:561-577.

2 Wehrman T, He X, Raab B, Dukipatti A, Blau H, Garcia KC. Structural and mechanistic insights into nerve growth factor interactions with the TrkA and p75 receptors. Neuron 2007; 53:25-38.

3 Hock CH, Heese K, Olivieri G, et al. Alterations in neurotrophins and neurotrophin receptors in Alzheimer's disease. J Neural Transm Suppl 2000; 59:171-174.

4 Zheng H, Koo EH. The amyloid precursor protein: beyond amyloid. Mol Neurodegener 2006; 1:5.

5 Greenfield JP, Gross RS, Gouras GK, Xu H. Cellular and molecular basis of beta-amyloid precursor protein metabolism. Front Biosci 2000; 5:D72-83.

6 Zou L, Wang Z, Shen L, et al. Receptor tyrosine kinases positively regulate BACE activity and Amyloid- $\beta$ production through enhancing BACE internalization. Cell Res 2007; 17:389-401.

7 Yan R, Han P, Miao H, Greengard P, Xu H. The transmembrane domain of the Alzheimer's $\beta$-secretase (BACE1) determines its late Golgi localization and access to APP substrate. J Biol Chem 2001; 276:36788-36796.

8 Walter J, Fluhrer R, Hartung B, et al. Phosphorylation regulates intracellular trafficking of $\beta$-secretase. J Biol Chem 2001; 276:14634-14641.

9 Wahle T, Prager K, Raffler N, Haass C, Famulok M, Walter J. GGA proteins regulate retrograde transport of BACE1 from endosomes to the trans-Golgi network. Mol Cell Neurosci 2005; 29:453-461.

10 Kins S, Lauther N, Szodorai A, Beyreuther K. Subcellular trafficking of the amyloid precursor protein gene family and its pathogenic role in Alzheimer's disease. Neurodegener Dis 2006; 3:218-226. 